\title{
A Distribution Method of High Precise Differential Corrections for a Network Beidou/RTK System Based on Vehicular Networks
}

\author{
Jian Liu ${ }^{1,2}$, Hongjiang Zheng ${ }^{1}$, Wei Chen ${ }^{1}$, Huiheng Liu ${ }^{3}$ \\ ${ }^{1}$ School of Information Engineering, WuHan University of Technology, WuHan 430070, Hubei, China \\ ${ }^{2}$ China Transport Telecommunications \& Information Center, BeiJing 100011, China \\ ${ }^{3}$ College of Applied Science, Jiangxi University of Science and Technology, Ganzhou 341000, Jiangxi, \\ China \\ Emails:liujian@cttic.cn hongjiangzheng@qq.com greatchen@whut.edu.cn lhh117@163.com
}

Abstract: In recent years urban traffic safety has become a widely concerned issue due to the increasing traffic accidents caused by the growth of private car ownership. A very promising method is to provide high precision navigation service to prevent accidents. Therefore, a Network BDS/RTK System Based on Vehicular Networks (NBRSBVNs) is proposed for high precision positioning in an urban complex traffic environment in this paper. This system considers comprehensively the characteristics of the vehicular network and network RTK techniques, and also provides a new distribution method of high precision differential correction information. According to the massive field tests, this system scheme can support real-time and online high precision navigation service of multiple users at the same time. It has important practical significance in applications for vehicles active safety in a complex urban environment.

Keywords: DSRC/WAVE, vehicular networks, network RTK technology, differential information distribution.

\section{Introduction}

The automobile has become an indispensable component of urban transportation. On the other hand, the growth of car ownership and the increase of vehicle speed are considered as major contributing factors in the increasing urban traffic accidents. Intelligent Transportation Systems (ITS) are designed to efficiently solve the increasing serious urban transport problems. The vehicular network is one of the core components of the system, which enables real-time services of information broadcasting, vehicle positioning and route navigation with the aid of a Global Navigation Satellite System (GNSS) [1]. Among these services, vehicle positioning 
is the key to support safety enhancement services, i.e., automatic collision avoidance and emergency vehicle notification.

Most of the GNSS, such as the Global Positioning System (GPS), Global Navigation Satellite System (GLONASS), Galileo and Beidou navigation Satellite system (BDS), are able to provide two types of positioning services with respect to its precision, one is the low-accuracy Open Service (OS), the other is the high accuracy Authorized Service (AS). On account of the high motion of the vehicles and the huge user capacity, the OS do not meet the demands of high-precise positioning of the vehicles in urban sites. However, the authorized service is expensive and restricted to a small group of users. Therefore, a differential positioning system is introduced to augment the open service, and improve the positioning accuracy, which includes the position, pseudo-range and carrier phase differential technologies. The latest network Real Time Kinematic (RTK) technique is developed from the carrier phase correction, which has better performance than the others.

The network RTK transmits phase corrections based on the massive carrier observables collected by Continuously Operating Reference Stations (CORS) to provide regional centimeter-level accuracy positioning services in real time [2]. The conventional data link which is suitable for surveying and mapping services employ mobile networks, i.e., the Global System for Mobile communication (GSM), the General Packet Radio Service (GPRS) and the Code Division Multiple Access (CDMA), because of their high availability and bandwidth, whereas a dedicated channel is needed for the transportation services on account of the real-time requirement. In this paper we propose an integration scheme of a Network BDS/RTK System Based on Vehicular Networks (NBRSBVNs) in order to provide accurate differential corrections to drivers. The notations and terminologies used in this paper are listed in Table 1.

Table 1. Notations and terminologies

\begin{tabular}{|l|l|}
\hline Notation and terminology & Description \\
\hline NBRSBVNs & Network BDS/RTK System Based on Vehicular Networks \\
\hline NRTK & Network real time kinematic \\
\hline BDS & Beidou navigation satellite system \\
\hline WSMP & Virtual reference station \\
\hline RS & WAVE short message protocol \\
\hline MS & Reference station \\
\hline SD & Mobile station \\
\hline DD & Single difference \\
\hline$k, l$ & Double difference \\
\hline$\lambda$ & Index number of the reference satellites \\
\hline$A, V$ & The carrier phase wavelength \\
\hline$T, I, O$ & The master reference station and the virtual reference station \\
\hline$N$ & The tropospheric errors, the ionospheric errors, and the ephemeris errors \\
\hline$m$ & The phase ambiguities \\
\hline$\varepsilon, e$ & The multipath errors \\
\hline$\Delta, \Delta \nabla$ & The noises in the carrier phase and code measurements \\
\hline$\varphi, P$ & The denotations of SD and DD \\
\hline$\rho$ & The carrier phase and code observations \\
\hline RSU & The geometric dilution of precision \\
\hline$\Delta d$ & Road Side Unit \\
\hline
\end{tabular}


The paper is organized as follows. Firstly, we introduce the characteristics of different wireless communication systems in Section 2. Then we discuss the composition and working process of a network RTK system, particularly, a Virtual Reference Station (VRS) system in Section 3. In Section 4 a scheme of NBRSBVNs and a new distribution method of high precision differential correction information are proposed. We verify the feasibility of the proposed scheme by a field testing in Section 5. Finally, we conclude the research and talk about its application prospects.

\section{Wireless communication system in a vehicular network}

In the vehicular network, the vehicles and roadside units are functioning as networking nodes, communicating among each other with useful information, such as safety warnings and traffic congestions [3]. With the aid of the vehicular communication system, the ITS is more efficient to avoid traffic accidents.

The IEEE Wireless Access in Vehicular Environments (WAVE) [4-6] is the international standard dedicated to a vehicular communication system, which is able to provide broadband communications with low time delays. The WAVE is the protocol suite standardized by IEEE in the IEEE 802.11p and IEEE 1609 protocol sets. The protocol layers used in this protocol suite are summarized as follows.

- IEEE 802.11p: Specifies the physical and MAC features required such that IEEE 802.11 could work in a vehicular environment.

- IEEE 802.2: Specifies the Logical Link Control (LLC).

- IEEE 1609.4: Provides a multi-channel operation that has to be added to IEEE 802.11p.

- IEEE 1609.3: Provides routing and addressing services required at the WAVE network layer by WAVE Short Message Protocol (WSMP).

- IEEE 1609.2: Specifies the WAVE security concepts of applications and management messages.

- IEEE 1609.1: Provides resource manager application of WAVE.

Table 2 shows the performance comparison of different wireless communication systems. According to the WAVE standard, the networking nodes are denoted as Dedicated Short-Range Communications (DSRC) devices, which are working within $5.9 \mathrm{GHz}$ band with a maximum range of $1000 \mathrm{~m}$. The WAVE also supports the Radio Technical Commission for Maritime (RTCM) protocols to transmit high-precision differential corrections.

Table 2. Performance comparison of different wireless communication systems

\begin{tabular}{|c|c|c|c|c|c|}
\hline Mode & Coverage & Transmission rate & Mobility & Security & Real-time \\
\hline GSM & $35-120 \mathrm{~km}$ & $\begin{array}{c}2.5 \mathrm{G}: 114 \mathrm{kbit} / \mathrm{s} \\
3 \mathrm{G}: 384 \mathrm{kbit} / \mathrm{s} \\
3.5 \mathrm{G}: 7.2 \mathrm{Mbit} / \mathrm{s}\end{array}$ & High & High & Mid \\
\hline WiMAX & $3-50 \mathrm{~km}$ & $70 \mathrm{Mbit} / \mathrm{s}$ & High & High & Low \\
\hline Wi-Fi & $10-100 \mathrm{~m}$ & $1-54 \mathrm{Mbit} / \mathrm{s}$ & Mid & Low & Mid \\
\hline DSRC/WAVE & $300-1000 \mathrm{~m}$ & $3-27 \mathrm{Mbit} / \mathrm{s}$ & High & Mid & High \\
\hline
\end{tabular}

142 


\section{Network RTK system}

Network RTK (NRTK) is developed from the traditional RTK technology, computer technology and networking technology. The idea of the network RTK is that a network of reference stations is established to share and transmit their observation data among each other. Then the data processing centre is able to mitigate the measurement errors based on the correction interpolation methods, which can provide the users with more precise RTK corrections. VRS system is the most popular solution to implement the network RTK, taking up almost $95 \%$ of the network RTK market.

\subsection{VRS system}

A typical VRS system is composed of Reference Stations (RS), a data processing/control centre, Mobile Stations (MS) and data links [7, 8], as shown in Fig. 1. Its working principle is as follows. First, the mobile station establishes connections to the RTK network and sends requests to the data processing/control centre. After the request is approved, a network of the available reference stations is organized by the control centre, in which the mobile station is considered as a virtual reference station. The reference stations with known coordinates transmit original observations to the data processing/control centre via the data links. Then the main computers are initialized to determine the ambiguity of the whole cycles of the observations and establish the combined error models simultaneously. When the data process is done, the corrections, i.e., the double differential corrections of observations, the ionospheric error corrections, the tropospheric error corrections, and etc., are transmitted to the mobile station. Eventually, the mobile station obtains high precision corrections to reduce the positioning errors.

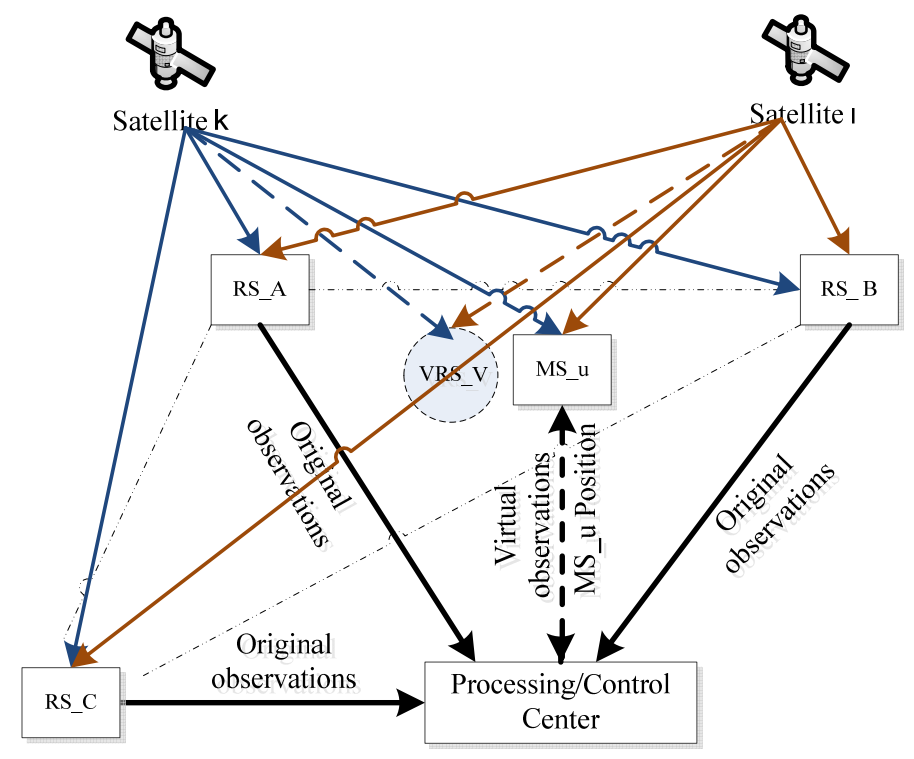

Fig. 1. System composition of VRS 


\subsection{Data processing method}

The Single Difference (SD) is the difference between the observations from two receivers, which can be used to eliminate the errors common for the two receivers. Double Difference (DD) is composed of a linear combination of either four individual observations or two single difference observations. By forming the double difference observations, it is possible to eliminate the inherent errors in the receivers and satellites, i.e., the receiver clock instability, satellite ephemeris errors and clock errors. In addition, most of the errors caused by the ionospheric delay and atmospheric refraction delay are prone to be removed with small baselines. Therefore, the network RTK employs double difference to compute the high precision corrections.

According to the schematic principle of the network VRS, shown in Fig. 1, given the circumstances of the two satellites $k$ and $l$, three reference stations $A$ (Master), $B$ and $C$, one virtual reference station $V$ and one mobile station $u$. The carrier phase and pseudorange observations can be given ( $k$ as reference satellite) as [9-12]:

$$
\begin{gathered}
\varphi_{V}^{l}=\varphi_{A}^{l}+\frac{1}{\lambda} \Delta \rho_{A V}^{l}+\frac{1}{\lambda}\left[-\Delta \nabla I_{A V}^{k l}+\Delta \nabla T_{A V}^{k l}+\Delta \nabla O_{A V}^{k l}+\Delta \nabla m_{A V \varphi}^{k l}+\varepsilon_{\Delta A V \varphi}\right], \\
P_{V}^{l}=P_{A}^{l}+\Delta \rho_{A V}^{l}+\Delta \nabla I_{A V}^{k l}+\Delta \nabla T_{A V}^{k l}+\Delta \nabla O_{A V}^{k l}+\Delta \nabla m_{A V_{P}}^{k l}+e_{\Delta A V_{P}} .
\end{gathered}
$$

Then the corresponding carrier phase and the pseudo-range observations of the reference satellite $k$ are taken:

$$
\begin{gathered}
\varphi_{V}^{l}=\varphi_{A}^{l}+\frac{1}{\lambda} \Delta \rho_{A V}^{l}, \\
P_{V}^{l}=P_{A}^{l}+\Delta \rho_{A V}^{l} .
\end{gathered}
$$

So the DD corrections of carrier phase observation equation and the pseudorange observation equations are respectively given as follows:

$$
\begin{aligned}
& \lambda \Delta \nabla \varphi_{A u}^{k l}+\lambda \Delta \nabla N_{V u}^{k l}=\Delta \nabla \rho_{A u}^{k l}+\underbrace{\left(-\Delta \nabla I_{A V}^{k l}+\Delta \nabla T_{A V}^{k l}+\Delta \nabla O_{A V}^{k l}\right)}_{\text {Corrections(known) }}+ \\
& +\underbrace{\left(-\Delta \nabla I_{V u}^{k l}+\Delta \nabla T_{V u}^{k l}+\Delta \nabla O_{V u}^{k l}+\Delta \nabla m_{V u \varphi}^{k l}+\varepsilon_{\Delta \nabla \varphi}\right)}_{\approx 0}, \\
& \Delta \nabla P_{V u}^{k l}=\Delta \nabla \rho_{A u}^{k l}+\underbrace{\left(\Delta \nabla I_{A V}^{k l}+\Delta \nabla T_{A V}^{k l}+\Delta \nabla O_{A V}^{k l}\right)}_{\text {Corrections(known) }}+ \\
& +\underbrace{\left(\Delta \nabla I_{V u}^{k l}+\Delta \nabla T_{V u}^{k l}+\Delta \nabla O_{V u}^{k l}+\Delta \nabla m_{V u P}^{k l}+e_{\Delta \nabla P}\right)}_{\approx 0},
\end{aligned}
$$

where $k$ and $l$ denote the satellites, $A$ and $V$ represent the reference stations, $\lambda$ is the carrier phase wavelength, $T$ stands for the tropospheric errors, $I$ stands for the ionospheric errors, $O$ denotes the ephemeris errors, $N$ - the phase ambiguities, $m$ - the multipath errors, $\varepsilon$ and $e$ represent the noises in the carrier phase and code measurements, $\Delta$ and $\Delta \nabla$ are the denotations of SD and DD respectively, $\varphi$ and $P$ are the carrier phase and code observables, $\rho$ is the geometric dilution of precision. Given that the distance from the mobile station to 
VRS station is less than $3 \mathrm{~km}$, we can obtain $\left(-\Delta \nabla I_{V u}^{k l}+\Delta \nabla T_{V u}^{k l}+\Delta \nabla O_{V u}^{k l}\right) \approx 0$, which implies that the VRS double difference over-performs the conventional RTK.

\section{NBRSBVNs}

Combining the vehicular network and the network RTK system, we propose a network BDS/RTK System Based on Vehicular Networks (NBRSBVNs), as well as a distribution method of high-precision difference corrections. The system is able to provide network RTK positioning services for mobile users in complex urban situations.

\subsection{Working principle of the system}

The NBRSBVNs consists of fixed reference stations, a data processing centre, RoadSide Units (RSU), mobile vehicles, and data communication links, as shown in Fig. 2. RSU and the vehicles are connected to the system via the vehicular network. Its basic principle is based on the real-time processing of the carrier phase/pseudorange of the mobile station and reference station, which must meet the requirements of the higher data transfer rate and reliability for the data transfer. The vehicular network provides three types of data links, namely, from the reference stations to the data processing centre, from the data processing centre to the road side units, from the road side units to the vehicles. The first and second types of data links require wired connections with high capacity and stability. The road side units can share the wireless WAVE networks with the vehicles.

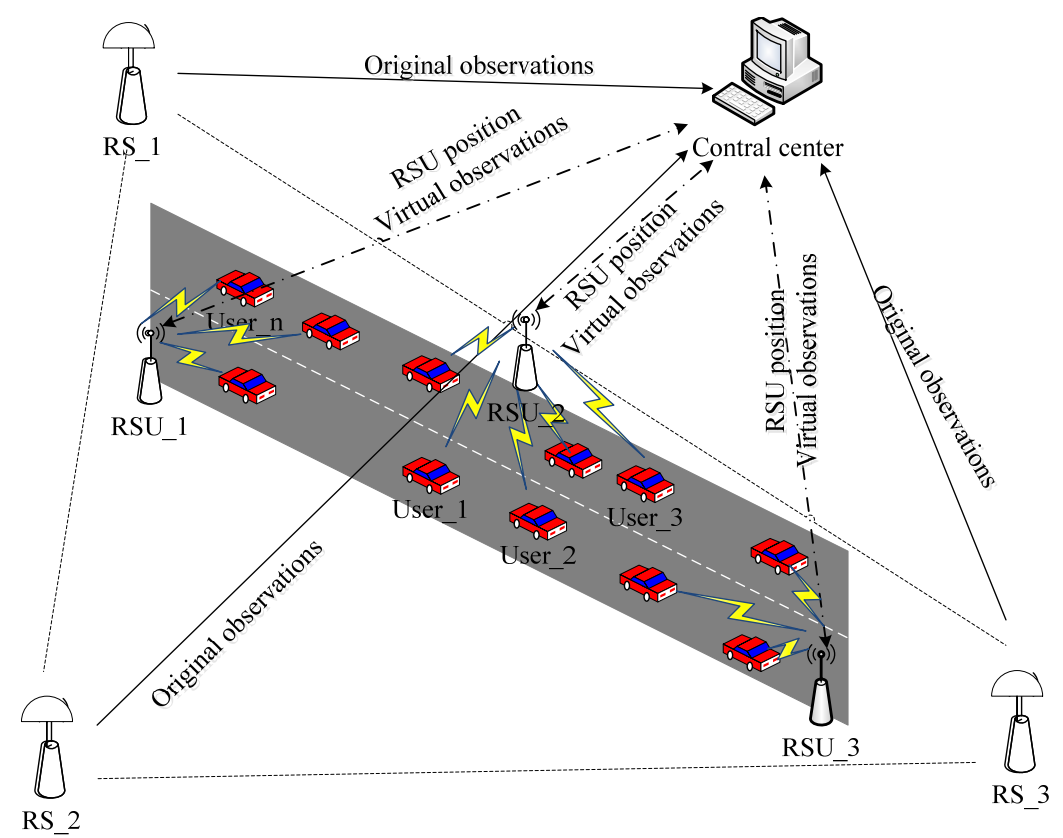

Fig. 2. The system structure diagram 


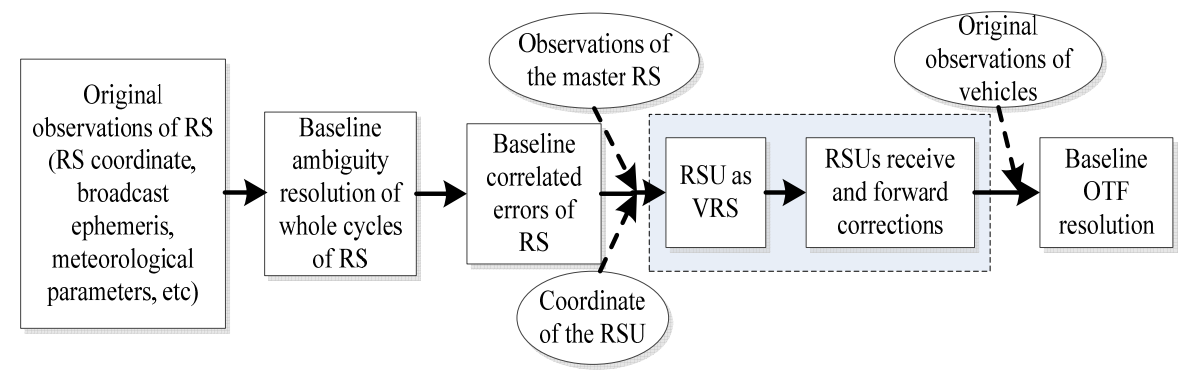

Fig. 3. The system working principle diagram

The work flow is similar to the conventional VRS system. The data processing centre computes the carrier phase ambiguities of the whole cycles based on the observations collected continuously by the reference stations and form the linear combination of double difference. However, the roadside units, i.e., the traffic lights and the electronic station boards are functioning as virtual reference stations to share their location with the data processing centre. The available reference stations in the vicinity of the road site unit are organized by the data processing centre to calculate the corrections to the location. Then the corrections are given as a feedback to the roadside units in RTCM messages. After having received corrections from the road side units via WAVE data links, the vehicles are able to implement high precision positioning. Fig. 3 shows the working principle.

\subsection{Difference corrections distribution}

In the NBRSBVNs, the correction data from the data processing center in the roadside unit are broadcasted by DSRC/WAVE, which involves a data format, the selection of transmission links and network protocols.

The quality of the positioning service relies on the real time message distribution. Many messaging protocols have been developed in industry for the dissemination of code-based and carrier-based corrections between reference stations and users. The most popular one is the messages developed by the RTCM study committee 104 (SC-104), which is referred to RTCM SC-104 messages. 63 types of messages are identified, which can be categorized into three states: fixed, tentative and reserved [13]. In our method we use six reserved messages, namely, from type 49 up to type 54, to contain Beidou messages. The carrier phase corrections and pseudo-range corrections are loaded in type 53 and 54 respectively, as shown in Table 3. Then the Beidou corrections are transmitted from the road side units to the vehicles through the vehicular network.

In addition, the Control CHannel $(\mathrm{CCH})$ is used for transmitting of corrections information, and the WAVE short message protocol (WSMP) is selected as the system network protocol.

Table 3. Definitions of Beidou message types

\begin{tabular}{|c|c|c|c|}
\hline Type & Content & Type & Content \\
\hline 49 & Parameters of the reference stations & 52 & Pseudo-range original observations \\
\hline 50 & Health information for satellites & 53 & Carrier phase corrections \\
\hline 51 & Carrier phase original observations & 54 & Pseudo-range corrections \\
\hline
\end{tabular}


Table 4. Locations of the test points and their static positioning horizontal error in the intersection

\begin{tabular}{|c|c|c|c|c|c|c|}
\hline \multirow{2}{*}{ Errors } & \multicolumn{5}{|c|}{ Test points and distances from the RSU $(\Delta d)$} \\
\cline { 2 - 7 } & & $\mathrm{T} 0(0 \mathrm{~m})$ & $\mathrm{T} 1(100 \mathrm{~m})$ & $\mathrm{T} 2(200 \mathrm{~m})$ & $\mathrm{T} 3(300 \mathrm{~m})$ & $\mathrm{T} 4(400 \mathrm{~m})$ \\
\hline \multirow{4}{*}{ East } & Mean & 0.000163 & 0.000162 & 0.000162 & 0.000167 & 0.000167 \\
\cline { 2 - 7 } & Max & 0.006916 & 0.006910 & 0.006920 & 0.006915 & 0.006916 \\
\cline { 2 - 7 } & Min & -0.006746 & -0.006746 & -0.006745 & -0.006756 & -0.006745 \\
\hline \multirow{4}{*}{ North } & Mean & 0.000575 & 0.000587 & 0.000575 & 0.000587 & 0.000586 \\
\cline { 2 - 8 } & Max & 0.009827 & 0.009827 & 0.009828 & 0.009827 & 0.009828 \\
\cline { 2 - 7 } & Min & -0.006633 & -0.006633 & -0.006632 & -0.006633 & -0.006630 \\
\hline \multirow{3}{*}{$\sqrt{\text { East }^{2}+\text { North }^{2}}$} & Mean & 0.002827 & 0.002848 & 0.002826 & 0.002839 & 0.002843 \\
\cline { 2 - 7 } & Max & 0.009844 & 0.009844 & 0.009846 & 0.009844 & 0.009844 \\
\cline { 2 - 7 } & Min & 0.000221 & 0.000201 & 0.000222 & 0.000220 & 0.000222 \\
\hline
\end{tabular}

\section{System testing and results analysis}

In order to assess the feasibility of the proposed NBRSBVNs in this paper, a static field test was carried out. In this work we consider that there are no high buildings in the case of signal transmission between RSU and mobile vehicles. Sinan M600 GNSS receivers were taken as a reference station and rover stations. First, the RSU of a cross road was measured as a virtual reference station. Then, one point in each direction of the cross road was measured. By real-time processing the measurements from both stations, epoch by epoch results are obtained. The results are shown in Table 4, Figs 4 and 5, where $\Delta d$ denotes the distance from the center. Compared with the measured locations, 5 points within one kilometre radius area, the positioning errors are trivial. The results show that our system is able to provide a high-precision Beidou positioning service.

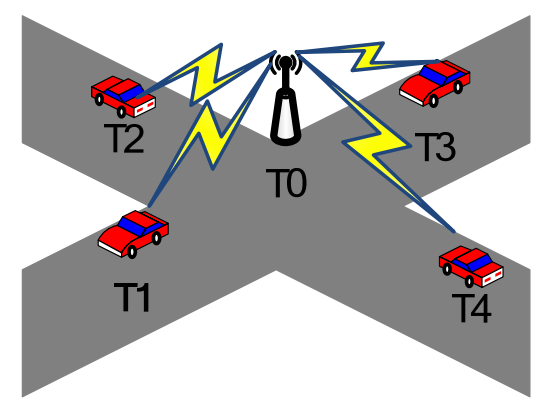

Fig. 4. The location distribution chart of test points in the intersection 

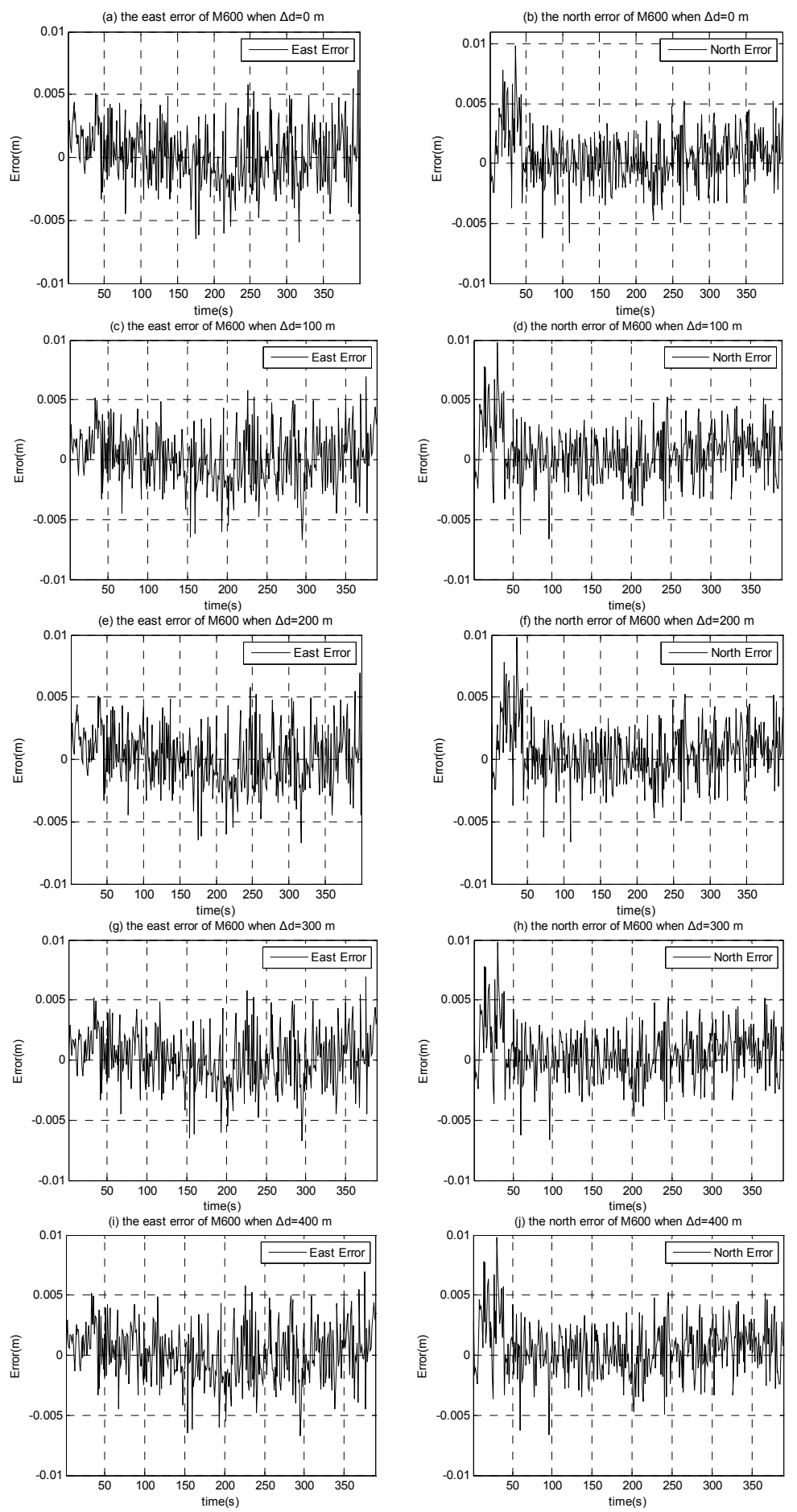

Fig. 5. Comparison of the static positioning horizontal error of 5 test points in the intersection 


\section{Conclusions}

The characteristics and working principles of the vehicular network and the network RTK system are introduced in this paper. Combining these two systems, it is able to provide high-precision positioning service for vehicles. The assessment results show that the positioning errors can be less than $1 \mathrm{~cm}$, meeting the requirements of the positioning service in complex urban situations.

Compared with the VRS system, the advantages of the NBRSBVNs are summarized as follows:

1. The positions of RSUs are fixed in this system. This makes the data processing center resolve differential information on demand, which can greatly reduce the computational effort.

2. This system has better real-time adaptability because it overcomes the initialization problem of the mobile terminals caused by changes of the VRS location in a network RTK system.

3. This system allows multiple mobile users to be online at the same time, without increasing of the calculated quantities.

Therefore, the scheme of the NBRSBVNs and the distribution method of corrections have important practical significance and application prospects for the high precision positioning of the vehicles in the urban traffic environment.

\section{References}

1. Karagiannis, G., O. Altintas, E. Ekici, G. Heijenk, B. Jarupan, K. Lin, T. W e il. Vehicular Networking: A Survey and Tutorial on Requirements, Architectures, Challenges, Standards and Solutions. - IEEE Communications Surveys \& Tutorials, Vol. 13, 2011, No 4, pp. 584-616.

2. T a n g, M. Evolutionary Placement of Continuously Operating Reference Stations of Network Real-Time Kinematic. -In: Proc. of 2012 IEEE World Congress on Computational Intelligence (WCCI), 1-8 June 2012

3. W a n g, Q., J. H u, J. Z h a n g. Performance Evaluation of Information Propagation in Vehicular Ad Hoc Network. - IET Intelligent Transport Systems, Vol. 6, 2012, No 2, pp. 187-196.

4. Morgan, Y. L. Notes on DSRC \& WAVE Standards Suite: Its Architecture, Design, and Characteristics. - IEEE Communications Surveys \& Tutorials, Vol. 12, 2010, No 4, pp. 504-518.

5. K e n n e y, J. B. Dedicated Short-Range Communications (DSRC) Standards in the United States. - Proceedings of the IEEE, Vol. 99, 2011, No 7, pp. 1162-1182.

6. U z c a t e g u i, R., G. A c o s t a-M a r u m. Wave: A Tutorial. - IEEE Communications Magazine, Vol. 47, 2009, No 5, pp. 126-133.

7. O dijk, D., P. J. G. T e u n is s e n. Improving the Speed of CORS Network RTK Ambiguity Resolution. - In: Proc. of 2010 IEEE/ION Position Location and Navigation Symposium (PLANS), May 2010, pp. 79-84.

8. A n s a ri, K., Y. F e n g, M. T a n g. A Runtime Integrity Monitoring Framework for Real-Time Relative Positioning Systems Based on GPS and DSRC. - IEEE Transactions on Intelligent Transportation Systems, 2014, No 99, pp. 1-13.

9. L i, C., D. H u a n g, L. Z h o u, R. X u. Improved Atmospheric Delay Mitigating Method for HighPrecision GNSS Positioning Based on Reference Station Network. - In: International Symposium on GPS/GNSS 2005, Hong Kong, 2005. 
10. C a i, Y., P. Ch e n g, X. M e n g, W. T a n g, C. S h i. Using Network RTK Corrections and LowCost GPS receiver for Precise Mass Market Positioning and Navigation Applications. In: Proc. of 2011 IEEE Intelligent Vehicles Symposium (IV), June 2011, pp. 345-349.

11. She pard, D. P., T. E. Hu m phreys. High-Precision Globally-Referenced Position and Attitude via a Fusion of Visual SLAM, Carrier-Phase-Based GPS, and Inertial Measurements. - In: Proc. of IEEE/ION Position, Location and Navigation Symposium (PLANS), May 2014, pp. 1309-1328.

12. L e i, B., Q. Li, X. L i. Landslide Monitoring System Based on RTK Technology. - In: Proc. of 4th International Workshop on Advanced Computational Intelligence, October 2011, pp. 525-528.

13. RTCM Special Committee No 104. RTCM Recommended Standards for Differential GNSS Version 2.3. - Radio Technical Commission for Maritime Services, 2001. 